\title{
HYPERSPECTRAL LASER-INDUCED FLUORESCENCE IMAGING FOR NONDESTRUCTIVE ASSESSING SOLUBLE SOLIDS CONTENT OF ORANGE
}

\author{
Muhua Liu ${ }^{1, *}$, Luring Zhang ${ }^{1}$, Enyou Guo ${ }^{1}$ \\ ${ }^{1}$ Engineering College, Jiangxi Agricultural University, Nanchang, China \\ * Corresponding author, address: Engineering College, Jiangxi Agricultural University, \\ Nanchang, 330045, P. R. China, Tel: 086-0791-3813260; Fax: 086-0791-3813260; Email: \\ suikelmh@sohu.com
}

Abstract: Laser-induced fluorescence imaging is a promising technique for assessing quality of fruit. This paper reports on using a hyperspectral laser-induced fluorescence imaging technique for measurement of laser-induced fluorescence from orange for predicting soluble solids content (SSC) of fruit. A laser $(632 \mathrm{~nm})$ was used as an excitation source for inducing fluorescence in oranges. Fluorescence scattering images were acquired from 'Nanfeng' orange and navel orange by a hyperspectral imaging system at the instance of laser illumination. Subsequent analysis of Fluorescence scattering images consisted in selecting regions of interest (ROIs) of $100 \times 50$ pixels, and ROIs were segment around the laser illumination point from Fluorescence scattering images. The hyperspectral fluorescence image data in the wavelength range of 700-1100 nm were represented by mean grey value of the ROIs. The fruit soluble solids content were measured using hand-held refractometer. A line regressing method was used for developing prediction models to predict fruit soluble solids content. Excellent predictions were obtained for soluble solids content with the correlation coefficient of prediction of 0.998 ('Nanfeng' orange) and 0.96 (navel orange). The results show that hyperspectral laser-induced fluorescence imaging is a very good method for nondestructive assessing soluble solids content of orange.

Keywords: hyperspectral imaging, laser-induced fluorescence, orange, soluble solids content

Liu, M., Zhang, L. and Guo, E., 2008, in IFIP International Federation for Information Processing, Volume 258; Computer and Computing Technologies in Agriculture, Vol. 1; Daoliang Li; (Boston: Springer), pp. 51-59. 


\section{INTRODUCTION}

Soluble solids content (or sugar index) is one of parameters for determining orange quality and maturity. Soluble solids content (SSC) may be determined from the juice extracted from fruit flesh using the refract metric method. This measurement method is destructive, inefficient and time consuming. A nondestructive sensing technique that is capable of measuring fruit quality parameters will be of great value in ensuring consistent quality fruit for the consumer. Many researches had been reported on the development of nondestructive sensing techniques for assessing soluble solids content of fruit. The technology and techniques include surface reflectance and transmittance of varying light energies. One good method is visible/near-infrared spectroscopy (VIS/NIRS). VIS/NIRS have became a non-destructive estimation of soluble solids content, oil contents, water content, dry matter content, acidity, firmness and others physiological properties of a number of fruit products indistinctly including citrus (Steuer et al., 2001); mandarin (Kawano et al., 1993; Miyamoto et al., 1997; McGlone et al., 2003; Antihus H. G. et al., 2006); and apple (Lu et al., 2000; McGlone et al., 2002; Park et al., 2003; Lu et al., 2005). However, spectroscopic assessment with relatively small point-source measurement have disadvantage compared to an imaging approach that characterizes the spatial variability of a sample material (Kim et al., 2001). Today, two recent techniques to access the quality of agricultural products are hyperspectral and mutispectral imaging. Hyperspectral and multispectral imaging is a relatively new technique for measuring the quality of food and agricultural products. The technique allows us to acquire both spectral and spatial information from a sample, thus offering some unique advantages over conventional imaging and spectroscopy techniques in detecting quality and safety of food and agricultural products (Lu et al., 1998). Latest research (Lu, 2003, 2004; Peng et al., 2005; Lu et al., 2006) showed that hyper- or multi-spectral scattering imaging, which measures light scattering and absorption, provides good assessment of fruit firmness. Several recent studies (Kim et al., 2003; Vargas et al., 2004; Ariana et al., 2006) reported that hyper- and/or multi-spectral fluorescence imaging is useful for detecting defects and safety of agricultural and food products.

In this research, a hyperspectral fluorescence imaging technique was used to measure laser-induced fluorescence scattering for assessing SSC quality of orange fruit. Specific objectives of this research were to:

- use a hyperspectral fluorescence imaging technique for acquiring fluorescence scattering images from orange fruit with the illumination of a laser of $632 \mathrm{~nm}$.

- develop line regressing method relating fluorescence spectral features to $S S C$ quality parameters of orange fruit. 


\section{MATERIALS AND METHODS}

\subsection{Orange samples}

Two hundred and sixty five 'Nanfen' oranges harvested from the orchard of Nanfeng country of Jiangxi province, P. R. China in 2006. Two hundred and sixty five navel oranges harvested from the orchard of Xinfeng country of Jiangxi province, P. R. China in 2006. The oranges were kept in dark at room temperature $\left(22^{\circ} \mathrm{C}\right)$ for at least $24 \mathrm{~h}$ before fluorescence and standard quality measurements were performed.

\subsection{Hyperspectral imaging system}

A hyperspectral fluorescence imaging system, schematically shown in Fig. 1, was assembled to acquire laser-induced fluorescence scattering images from orange fruit. The system consisted of a hyperspectral imaging unit, a $632 \mathrm{~nm}$ laser unit, a fruit holder, and the imaging chamber. The hyperspectral imaging unit was composed of a high sensitivity back-thinned charge coupled device (CCD) camera, an imaging spectrograph (ImSpector V10E, Spectral Imaging Ltd., Oulu, Finland), and a computer used to control the CCD camera and acquire images. The laser unit was equipped with a laser drive and temperature control, and its $632 \mathrm{~nm}$ laser was to generate a circular beam of $1.5 \mathrm{~mm}$ diameter at the fruit. As the laser beam hit the fruit, a portion of the monochromatic light was absorbed by the fruit tissue with subsequent release of fluorescence (i.e., light of longer wavelengths). This resulted in a fluorescence emission distribution image around the illumination point at the surface of the fruit. The imaging spectrograph line scanned the fruit to collect the fluorescence scattering image at the surface of the fruit. As the light passed through the imaging spectrograph, it was dispersed into different wavelengths while its spatial information was preserved. The dispersed light signals were then projected onto the CCD detector, creating a two-dimensional image: one dimension represents the spatial and the other dimension spectral. The line scanning position of the imaging spectrograph was $2 \mathrm{~mm}$ off from the beam incident center to avoid saturation caused by high intensity signals. The hyperspectral imaging system had an effective spectral region of $408-1117 \mathrm{~nm}$ with a nominal spectral resolution of $2.8 \mathrm{~nm}$ and a spatial resolution of $0.2 \mathrm{~mm} /$ pixel (Fig. 2). 


\subsection{Fluorescence imaging acquiring and fruit quality measurement}

\subsubsection{Fluorescence imaging acquiring}

Images were collected in a dark room with only the laser light source. Fluorescence measurements were first performed on individual orange fruit, followed by standard measurements for SSC. Each orange with the stemcalyx end horizontal was placed on the plate which was drive by motor in the imaging chamber, which was completely shielded from ambient light during the measurement (Fig. 1). The hyperspectral imaging system captured the fluorescence scattering image of orange, and these images were saved in a computer for further analysis.

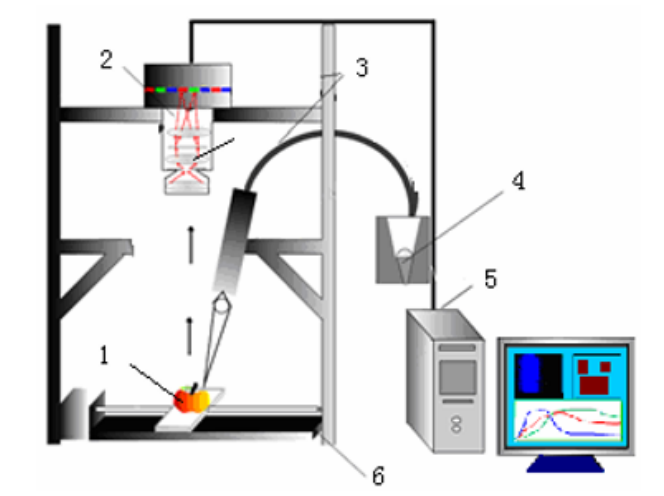

1.orange, 2.CCD camera, 3.fiber, 4.laser, 5.computer

Fig. 1. Hyperspectral imaging system

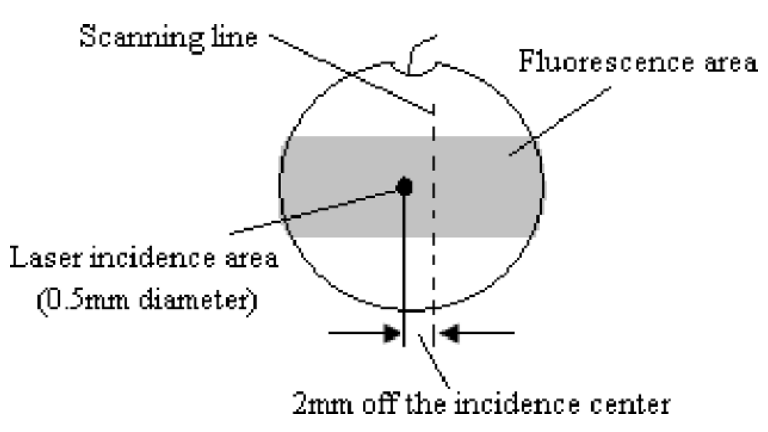

Fig. 2. The line scanning position of the hyperspetral imaging system, the laser incidence center and fluorescence area 


\subsubsection{Orange SSC measurement}

Soluble solids content expressed in Brix was measured from the juice released using a hand-held refractometer (Model WZ-103, Zhongyou Optical Instrument Corp., China).

\subsection{Fruit quality prediction models}

A typical hyperspectral fluorescence image is displayed in Fig. 3. Each scattering image is composed of hundreds of spectra with each coming from a different position at the fruit surface. Subsequent analysis of Fluorescence scattering images consisted in selecting regions of interest (ROIs) of $100 \times 50$ pixels, and ROIs were segment around the laser illumination point track from Fluorescence scattering images. To properly characterize each scattering image, mean of CCD count was calculated from ROIs of the spatial scattering profiles $(100 \times 50$ pixels $)$ of the hyperspectral image for each wavelength from 700 to $1100 \mathrm{~nm}$. This mean value calculated from ROIs was then assumed to represent the fluorescence intensity of fruit images.

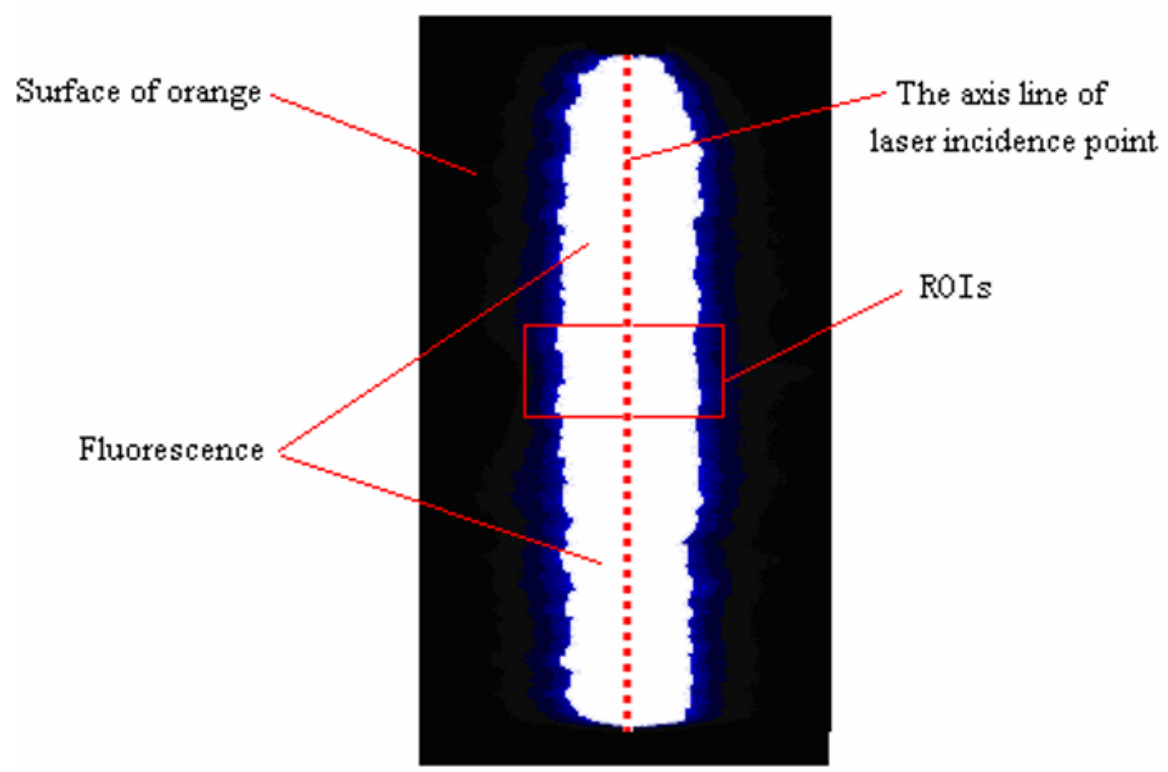

Fig. 3. Hyperspectral fluorescence image from a "Nanfeng" orange

A line regressing method and combining principal component (PC) analysis was proposed for developing orange quality prediction models. Principal component analysis provides an effective means for analysis 
essential spectral region. As such, PC analysis was performed on mean spectra of hyperspectral fluorescence image. PC scores and coefficients of mean spectra were used to select spectral region. Then line regressing method to develop a calibration model for predicting orange SSC. The procedure of developing and validating the prediction model for each fruit quality parameter is demonstrated through the algorithm for orange fruit SSC prediction.

First, all samples were arranged in ascending order for their $S S C$ values. Then samples were then divided into the training groups (200 samples) and validation groups (60 samples). The training samples were analyses using principal component (PC) for look for effective spectral region. 920-992 nm and 949-1000 $\mathrm{nm}$ were respectively thought as best spectral region for 'Nanfeng' orange and navel orange. Then the SSC prediction model was developed using line regressing method. Finally, the obtained model was used to predict orange $S S C$ for the validation samples. This procedure was performed using programmer file created in Mathlab (The Math Works Inc., Natick, MA, USA). In this paper, we only present the results from the best prediction model, as measured by $R$ and the standard error of prediction (SEP).

\section{RESULTS AND DISCUSSION}

\subsection{The statistics of orange SSC}

The statistics of fruit SSC for all oranges are summarized in Table 1. The $S S C$ distribution of the oranges was in the range from 12.6 to $20.93^{\circ}$ brix ('Nanfeng' orange) and 10.8 to $16^{\circ}$ brix (navel orange).

Table 1. statistics of orange SSC measured by standard method

\begin{tabular}{lcccc}
\hline Sample & Maximum & Minimum & Mean & Standard deviation \\
\hline 'Nanfeng' orange & 20.93 & 12.6 & 16.32 & 2.04 \\
Navel orange & 16 & 10.8 & 13.06 & 1.05 \\
\hline
\end{tabular}

\subsection{Spectra of orange}

Spectra of orange scattering image was characterized by the mean of intensity CCD count that calculated from The ROIs of the spatial scattering profiles $(100 \times 50$ pixels $)$ of the hyperspectral image for each wavelength from 700 to $1100 \mathrm{~nm}$. Fig. 4 shows the spectra of one 'Nanfeng' orange and the result was same for navel orange. It shows that laser-induced 
fluorescence was characterized by period of stranger and weak intensity, and the wavelength interval was about $20 \mathrm{~nm}$.

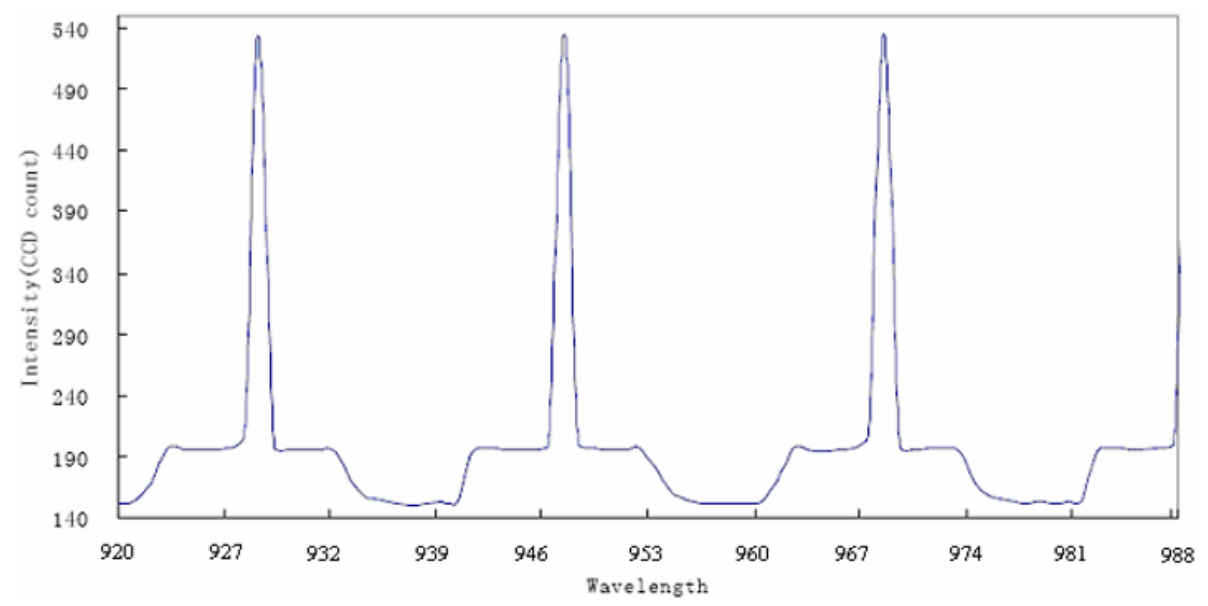

Fig. 4. The mean of CCD intensity for one 'Nanfeng' orange in 920-992 nm

\subsection{Hyperspectral laser-induced fluorescence prediction models}

After analyzing, spectra data of 920-992 nm ('Nanfeng' orange) and 949-1000 nm (navel orange) were respectively selected for building models. The fruit SSC prediction results from fluorescence imaging are shown in Table 2. The fluorescence prediction of fruit SSC found in this research is sufficient for sorting and grading oranges. An excellent correlation was obtained between fluorescence measurement and fruit SSC, The $R$ and SEP were $0.998,0.029$ for 'Nanfeng' orange and $0.96,0.28$ for navel orange. The SSC prediction results by the fluorescence prediction model are considerably good than those obtained using visible/shortwave NIR spectroscopy in the wavelengths of $500-1100 \mathrm{~nm}$.

Table 2. prediction model results for SSC

\begin{tabular}{lcc}
\hline Sample & The correlation coefficient (R) & The standard error of prediction (SEP) \\
\hline 'Nanfeng' orange & 0.998 & 0.029 \\
Navel orange & 0.96 & 0.28 \\
\hline
\end{tabular}

\section{CONCLUSIONS}

Past research show that VIS/NIR spectroscopy is a suitable tool for measuring of fruit SSC. A new method based on Hyperspectral laser-induced 
fluorescence imaging was used to nondestructive measuring fruit SSC in this study.

The Hyperspectral laser-induced fluorescence images can be used for fruit SSC prediction with small prediction errors over the range of 20.93 to 10.8 of SSC. It had good predictions of SSC with values for the correlation coefficient $\mathrm{R}=0.998$ for 'nanfeng' orange and $\mathrm{R}=0.96$ for navel orange, and sufficient for sorting and grading oranges.

The experiment results show that hyperspectral fluorescence imaging technique is potentially useful for assessing SSC of orange fruit. However, advance researching in fluorescence measurement and data analysis are needed in order to predicting the color, firmness and contamination of orange quality.

\section{ACKNOWLEDGMENTS}

The authors appreciate the National Natural Science Foundation of China for assistance.

\section{REFERENCES}

Antihus, H.G., He, Y., \& Pereira, G.A. Non-destructive measurement of acidity, soluble solids and firmness of Satsuma mandarin using Vis/NIR-spectroscopy techniques. Journal of Food Engineering, 2006, 77, 313-319.

Ariana, D.P., Shrestha, B.P., \& Guyer, D.E. Integrating reflectance and fluorescence imaging for apple disorder classification. Comput. Electron. Agric. 2006, 50, 148-161.

DeEll, J.R., Kooten, O., Prange, R.K., \& Murr, D.P. Applications of chlorophyll fluorescence techniques in postharvest physiology. Horticultural Reviews, 1999, 23, 69-107.

Kawano, S., Fujiwara, T., \& Iwamoto, M.J. Nondestructive determination of sugar content in Satsuma mandarin using near infrared (NIR) transmittance. Journal Japanese Society Horticultural Science, 1993, 62 (2), 465-470.

Kim, M.S., Chen, Y.R., \& Mehl, P.M. Hyperspectral reflectance and fluorescence imaging system for food quality and safety. Transactions of the ASAE. 2001, 44(3), 721-729.

Kim, M.S., Lefcourt, A.M., \& Chen, Y.R. Multispectral laser-induced fluorescence imaging system for large biological samples. Appl. Opt. 2003, 42, 3927-3934.

$\mathrm{Lu}, \mathrm{R}$. Imaging spectroscopy for assessing internal quality of apple fruit. ASAE Paper No. 036012. 2003 ASAE, St. Joseph, MI.

$\mathrm{Lu}, \mathrm{R}$. Multispectral imaging for predicting firmness and soluble solids content of apple fruit. Postharvest Biology and Technology, 2004, 31, 147-157.

$\mathrm{Lu}, \mathrm{R}$., Bailey, B.B. NIR measurement of apple fruit soluble solids content and firmness as affected by postharvest storage. ASAE Paper No. 056070. 2005, ASAE, St. Joseph, MI.

Lu, R., Chen, Y.R. Hyperspectral imaging for safety inspection of food and agricultural products. SPIE Proc. 1998, 3544, 121-133. 
Lu, R., Guyer, D.E., \& Beaudry, R.M. Determination of firmness and sugar content of apples using near-infrared diffuse reflectance. J. Texture Stud. 2000, 31, 615-630.

McGlone, V.A., Fraser, D.G., Jordan, R.B., \& Kunnemeyer, R. Internal quality assessment of mandarin fruit by Vis/NIR spectroscopy. Journal of Near Infrared Spectroscopy, 2003, 11, 323-332.

McGlone, V.A., Jordan, R.B., \& Martinen, P.J. Vis/NIR estimation at harvest of pre- and post-storage quality indices for 'Royal Gala' apple. Postharvest Biology and Technology, 2002, 25, 135-144.

Miyamoto, K., Kawauchi, M., \& Fukuda, T. Classification of high acid fruits by PLS using the near infrared transmittance (NIT) spectra of intact Satsuma mandarins. Journal of Near Infrared Spectroscopy, 1997, 6 (1-4), 267-271.

Park, B., Abbott, J.A., Lee, K.J., Choi, C.H., \& Choi K.H. Near-infrared diffuse reflectance for quantitative and qualitative measurement of soluble solids and firmness of delicious and gala apples. Trans. ASAE, 2003, 46, 1721-1731.

Peng, Y., Lu, R. Modeling multispectral scattering profiles for prediction of apple fruit firmness. Trans. ASAE, 2005, 48, 235-242.

Steuer, B., Schulz, H., \& Lager, E. Classification and analysis of citrus oils by NIR spectroscopy. Food Chemistry, 2001, 72, 113-117.

Vargas, A.M., Kim, M.S., Tao, Y., Lefcourt, A., \& Chen, Y.R. Safety inspection of cantaloupes and strawberries using multispectral fluorescence imaging techniques. ASAE Paper No. 043056. 2004, ASAE, St. Joseph, MI. 